\title{
Hybrid Analog-Digital Millimeter-Wave MU-MIMO Transmission With Virtual Path Selection
}

\author{
Ang Li, Student Member, IEEE, and Christos Masouros, Senior Member, IEEE
}

\begin{abstract}
In this letter, we propose a low-complexity hybrid precoding and combining design for the millimeterwave MU-MIMO transmission, applicable to both fully connected and sub-connected structures. Analog precoding and combining schemes are first designed, where a joint approach, a decoupled approach, and a sub-optimal approach are proposed to harvest the array gain. Virtual path selection is performed to maximize the channel gain of the analog effective channel. Then, based on the effective channel, a low-dimensional zero-forcing precoding is applied in the baseband to manage the interference. The simulation results show that the proposed techniques offer an enhanced performance-complexity tradeoff compared with both existing hybrid schemes and fully digital schemes.
\end{abstract}

Index Terms-mmWave-MIMO, hybrid design, precoding, combining.

\section{INTRODUCTION}

$\mathbf{M}$ ILLIMETER-WAVE (mmWave) communications have emerged as one of the most promising techniques for the future fifth-generation $(5 \mathrm{G})$ cellular systems to exploit the underutilized mmWave spectrum resources [1], [2]. To overcome the pathloss encountered in the mmWave frequencies and provide reasonable signal-to-noise-ratio (SNR), mmWave systems will require a large number of antennas at the base station (BS) [1]. The small carrier wavelength of mmWave communications enables packing a large number of antennas in a small physical space [2]. For a large antenna system, simple linear precoding schemes, such as zero-forcing (ZF), can achieve near-optimal performance [3]. Nevertheless, for conventional digital precoding schemes to be applied, each antenna needs to be connected to a dedicated radio frequency (RF) chain, which becomes impractical in the large scale MIMO regime due to the high cost and power consumption.

Due to the above practical constraints, analog processing or RF precoding schemes have been designed with lowcost phase shifters for current mmWave systems [4], [5]. Recently, hybrid precoding and combining designs for singleuser mmWave systems have been proposed [6], [7], where the processing is divided into the analog and digital domain. In [6], an orthogonal match pursuit (OMP) algorithm is proposed to obtain the analog and baseband precoding matrix, while an iterative hybrid scheme is proposed in [7]. The extensions

Manuscript received September 9, 2016; revised October 11, 2016; accepted October 23, 2016. Date of publication October 26, 2016; date of current version February 9, 2017. This work was supported by the Royal Academy of Engineering, UK, the Engineering and Physical Sciences Research Council (EPSRC) project EP/M014150/1, and the China Scholarship Coucil (CSC). The associate editor coordinating the review of this letter and approving it for publication was L. Wang.

The authors are with the Department of Electronic and Electrical Engineering, University College London, London, WC1E 7JE, U.K. (e-mail: ang.li.14@ucl.ac.uk; chris.masouros@ieee.org).

Digital Object Identifier 10.1109/LCOMM.2016.2621741 to multi-user case have been designed in [8]-[10], where a codebook-based design is proposed in [8], while in [9] a minimum mean-squared error (MMSE) approach is applied upon the OMP approach. We note that the above designs for mmWave systems all assume a fully-connected structure, where each antenna is connected to all RF chains. However, such structure requires a large number of switches and phase shifters, which introduce significant insertion losses and degrade the resulting energy efficiency [11]. Therefore, a subconnected structure where each antenna is only connected to one RF chain is promising for practical implementation.

In this letter, we consider the multi-user transmission and propose a low-complexity hybrid design, which applies to both fully-connected structures and sub-connected structures. Three distinct designs are proposed respectively, where we perform virtual path selection in the RF domain to maximize the effective channel gain. Then, based on the lowdimensional effective analog channel, ZF precoding is applied in the baseband to eliminate the multi-user interference, which can approach the performance of digital precoding schemes, for a reduced hardware complexity. Our complexity analysis and simulation results validate the performance-complexity advantages of the proposed schemes over existing techniques, and show that sub-connected structures can achieve a much higher energy efficiency than fully-connected structures.

\section{Hybrid ANAlog-Digital TRAnSmission Model}

We consider a MU-MIMO downlink system, where a BS with $N_{t}$ antennas and $N_{R F}^{t}$ RF chains communicates with $K$ mobile stations (MSs), each equipped with $N_{r}^{k}$ antennas. Following [8], we focus on the case where the BS employs $K$ RF chains to support the transmission of $K$ streams, i.e. $K=N_{R F}^{t}$. Considering hybrid precoding, the transmitted signal can be expressed as

$$
\mathbf{x}=\mathbf{F}_{R F} \mathbf{F}_{B B} \mathbf{S},
$$

where $\mathbf{F}_{B B}=\left[\mathbf{f}_{B B_{1}}, \mathbf{f}_{B B_{2}}, \ldots, \mathbf{f}_{B B_{K}}\right] \in C^{K \times K}$ is the baseband precoder. $\mathbf{s} \in C^{K \times 1}$ is the transmitted symbol vector and $\mathrm{E}\left\{\mathbf{s s}^{H}\right\}=\frac{P}{K} \cdot \mathbf{I}$, where $P$ is the average total transmit power and we assume equal power allocation for each stream. $\mathbf{F}_{R F} \in C^{N_{t} \times K}$ is the RF precoder implemented with phase shifters, and therefore each entry of $\mathbf{F}_{R F}$ is of constant modulus. When fully-connected structures are considered as shown in Fig. 1 (a), $\mathbf{F}_{R F}=\left[\mathbf{f}_{1}^{\text {full }}, \mathbf{f}_{2}^{\text {full }}, \ldots, \mathbf{f}_{K}^{\text {full }}\right]$ is a full matrix with all elements being non-zero and normalized to satisfy $\left|\mathbf{f}_{k}^{\text {full }}(m)\right|^{2}=\frac{1}{N_{t}}$. For sub-connected structures as shown in Fig. 1 (b), $\mathbf{F}_{R F}=\operatorname{diag}\left[\mathbf{f}_{1}^{s u b}, \mathbf{f}_{2}^{s u b}, \ldots, \mathbf{f}_{K}^{\text {sub }}\right]$ becomes a block diagonal matrix, and each entry satisfies 


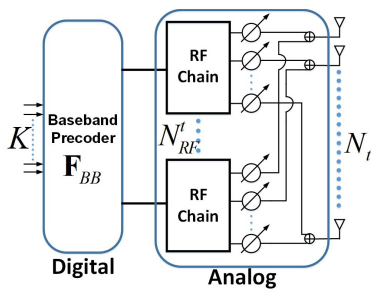

(a) Fully-connected structure

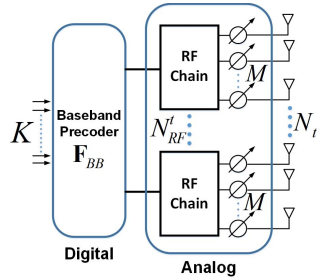

(b) Sub-connected structure
Fig. 1. Transmit antenna array structure.

$\left|\mathbf{f}_{k}^{s u b}(m)\right|^{2}=\frac{1}{M}$, where $M$ is the number of antennas connected to each RF chain. The total power constraint is satisfied by normalizing $\mathbf{F}_{B B}$ such that $\left\|\mathbf{F}_{R F} \mathbf{F}_{B B}\right\|_{F}^{2}=K$ [8]. Each MS $k$ is equipped with one RF chain, and a RF only combiner $\mathbf{w}_{k}^{H} \in C^{1 \times N_{r}^{k}}$ is applied, where each entry of the vector $\mathbf{w}_{k}$ is of constant modulus, normalized to satisfy $\left|\mathbf{w}_{k}(m)\right|^{2}=\frac{1}{N_{r}^{k}}$. Then, the received symbol for MS $k$ can be expressed as

$$
r_{k}=\mathbf{w}_{k}^{H} \mathbf{H}_{k} \mathbf{F}_{R F} \mathbf{f}_{B B_{k}} s_{k}+\mathbf{w}_{k}^{H} \mathbf{H}_{k} \mathbf{F}_{R F} \sum_{j \neq k} \mathbf{f}_{B B_{j}} s_{j}+\mathbf{w}_{k}^{H} \mathbf{n}_{k},
$$

where $\mathbf{H}_{k} \in C^{N_{r}^{k} \times N_{t}}$ is the mmWave channel from the BS to MS $k, \mathbf{n}_{k}$ is the additive noise vector assumed to be circular symmetric Gaussian with zero mean and covariance $\sigma^{2} \mathbf{I}$.

\section{Proposed Hybrid Schemes}

In this section the proposed schemes are introduced, where we apply phase-only weights for the analog precoder and combiner. The analog domain is firstly designed, followed by the design in the digital domain. We first consider the fully-connected structures, and then extend to sub-connected structures. By denoting $\mathbf{W}=\operatorname{diag}\left[\mathbf{w}_{1}, \ldots, \mathbf{w}_{K}\right]$ and $\mathbf{H}=$ $\left[\mathbf{H}_{1}^{T}, \mathbf{H}_{2}^{T}, \ldots, \mathbf{H}_{K}^{T}\right]^{T}$, we can express the effective RF channel in a compact form as

$$
\tilde{\mathbf{H}}=\mathbf{W}^{H} \mathbf{H F}_{R F} .
$$

Note that the diagonal entries in $\tilde{\mathbf{H}}$ are the RF-to-RF channel gains, while the off-diagonal terms denote interference. Therefore, we focus on the diagonal terms of the effective channel, while the off-diagonal interference can be handled by the low-dimensional baseband precoder. Then, the $k$ th diagonal entry of $\tilde{\mathbf{H}}$ can be expressed as

$$
\tilde{\mathbf{H}}(k, k)=\mathbf{w}_{k}^{H} \mathbf{H}_{k} \mathbf{f}_{k}^{\text {full }} .
$$

The aim of the proposed scheme is to extract the phases of the downlink channel from the BS to MSs to formulate the analog precoder and combiner, where we employ the conjugate transpose to maximize the analog effective channel gain. We note that in a MU-MIMO system, for each user $k$, $\mathbf{H}_{k} \in C^{N_{r}^{k} \times N_{t}}$, while $\mathbf{f}_{k}^{\text {full }} \in C^{N_{t} \times 1}$. Therefore, direct conjugate transpose cannot be applied. We then perform a virtual path selection scheme to maximize $\tilde{\mathbf{H}}(k, k)$ for each user $k$.

\section{A. Joint Precoding and Combining Design}

In the joint design, for each user $k$ we propose to jointly design $\mathbf{w}_{k}$ and $\mathbf{f}_{k}^{\text {full }}$. As $\mathbf{H}_{k}$ is $N_{r}^{k} \times N_{t}$ dimensional,

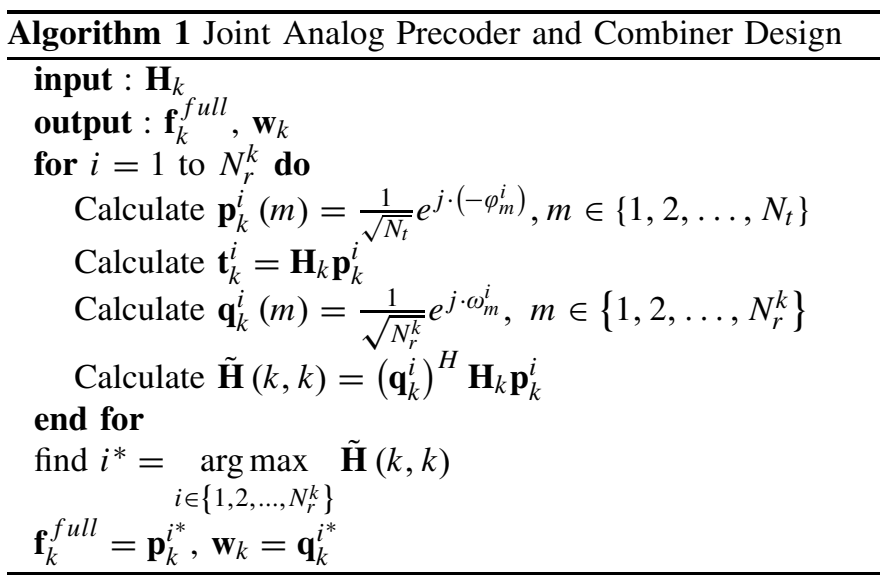

we decompose it into $\mathbf{H}_{k}=\left[\left(\mathbf{h}_{k}^{1}\right)^{T},\left(\mathbf{h}_{k}^{2}\right)^{T}, \ldots,\left(\mathbf{h}_{k}^{N_{r}^{k}}\right)^{T}\right]^{T}$ and each $\mathbf{h}_{k}^{i}$ can represent a virtual path. In the first step, for each $i \in\left\{1,2, \ldots, N_{r}^{k}\right\}$, we design a temporary analog precoder $\mathbf{p}_{k}^{i}$ as the normalized conjugate transpose of $\mathbf{h}_{k}^{i}$, given by [10]

$$
\mathbf{p}_{k}^{i}(m)=\frac{1}{\sqrt{N_{t}}} e^{j \cdot\left(-\varphi_{m}^{i}\right)}, \quad m \in\left\{1,2, \ldots, N_{t}\right\},
$$

where $\varphi_{m}^{i}$ is the angle of the $m$ th element in $\mathbf{h}_{k}^{i}$. Then, a temporary analog combiner $\mathbf{q}_{k}$ is similarly designed as

$$
\mathbf{q}_{k}^{i}(m)=\frac{1}{\sqrt{N_{r}^{k}}} e^{j \cdot \omega_{m}^{i}}, \quad m \in\left\{1,2, \ldots, N_{r}^{k}\right\},
$$

where $\omega_{m}^{i}$ denotes the angle of the $m$ th element in $\mathbf{t}_{k}^{i}=\mathbf{H}_{k} \mathbf{p}_{k}^{i}$. In the second step, we select the virtual path $i$ that achieves the largest effective channel gain $\tilde{\mathbf{H}}(k, k)$, expressed as

$$
\left(i^{*}, \mathbf{p}_{k}^{i^{*}}, \mathbf{q}_{k}^{i^{*}}\right)=\underset{i \in\left\{1,2, \ldots, N_{r}^{k}\right\}}{\arg \max }\left[\left(\mathbf{q}_{k}^{i}\right)^{H} \mathbf{H}_{k} \mathbf{p}_{k}^{i}\right] .
$$

The detailed joint scheme is given in Algorithm 1.

For user $k$, each time the proposed scheme employs a specific row of $\mathbf{H}_{k}$ to obtain $\mathbf{p}_{k}^{i}$ and $\mathbf{q}_{k}^{i}$, while the selected $\mathbf{f}_{k}^{\text {full }}$ that maximizes the effective channel gain $\tilde{\mathbf{H}}(k, k)$ applies to all rows of $\mathbf{H}_{k}$ at the BS for the $k$ th user. At each MS, all the receive antennas are active and apply the analog combiner $\mathbf{w}_{k}$ to obtain the received symbol for demodulation. Therefore, the proposed scheme is not an antenna selection scheme but termed as a 'virtual path selection' scheme. Furthermore, we note that the design of analog precoder and combiner is only dependent on the channel, and therefore given the channel knowledge no additional information exchange is required between the BS and MSs for the proposed schemes.

\section{B. Decoupled Precoding and Combining Design}

For the proposed joint design, $\mathbf{F}_{R F}$ and $\mathbf{W}$ need to be calculated at the BS, while each user $k$ needs to calculate $\mathbf{F}_{R F}$ and $\mathbf{w}_{k}$. In this section, we introduce a decoupled scheme, where the BS only needs to calculate $\mathbf{F}_{R F}$, while the calculation of each $\mathbf{w}_{k}$ is solely conducted at each MS. 
Firstly, for each user $k, \mathbf{p}_{k}^{i}, i \in\left\{1,2, \ldots, N_{r}^{k}\right\}$ is formulated by (5), then we find $i^{*}$ that maximizes $\mathbf{h}_{k}^{i} \mathbf{p}_{k}^{i}$, expressed as

$$
\left(i^{*}, \mathbf{p}_{k}^{i^{*}}\right)=\underset{i \in\left\{1,2, \ldots, N_{r}^{k}\right\}}{\arg \max } \mathbf{h}_{k}^{i} \mathbf{p}_{k}^{i},
$$

which is equivalent to finding $\mathbf{h}_{k}^{i}$ with the largest norm. For each analog combiner, $\mathbf{w}_{k}=\mathbf{q}_{k}$ is then obtained by (6) based on $\mathbf{t}_{k}^{*}=\mathbf{H}_{k} \mathbf{p}_{k}^{i^{*}}$. The detailed algorithm is given below.

\section{Sub-Optimal Precoding and Combining Design}

A low-complexity scheme is further proposed in this subsection. Without loss of generality, for each user $k$, we employ $\mathbf{h}_{k}^{1}$ to obtain $\mathbf{f}_{k}$ and $\mathbf{w}_{k}$, which saves the complexity of calculating the optimal $i^{*}$. The algorithm is given in Algorithm 3.

\section{Case for Sub-Connected Structures}

When sub-connected structures are considered, each $\mathbf{f}_{k}^{\text {sub }} \in$ $C^{M \times 1}$. Then, we design each $\mathbf{p}_{k}^{i}$ as the conjugate transpose of part of the $\mathbf{h}_{k}^{i}$, similarly as in (5), where for sub-connected structures, we have

$$
\mathbf{p}_{k}^{i}(m)=\frac{1}{\sqrt{N_{t}}} e^{j \cdot\left(-\varphi_{m}^{i}\right)}, \quad m \in\{(k-1) M+1, k M\} .
$$

Then, the final analog precoder $\mathbf{f}_{k}^{s u b}$ and combiner $\mathbf{w}_{k}$ for subconnected structures can be similarly obtained for each of the above schemes. The analog precoder for sub-connected structures can only harvest part of the array gains, and therefore a performance gap in terms of sum capacity will be observed compared to fully-connected structures.

\section{E. Baseband Design}

With $\mathbf{F}_{R F}$ and $\mathbf{W}$ obtained based on the above designs that focus on the analog effective channel gains in the diagonal, at the baseband we employ ZF precoding to eliminate the multi-user interference in the off-diagonal of $\tilde{\mathbf{H}}$, expressed as

$$
\mathbf{F}_{B B}=f \cdot \tilde{\mathbf{H}}^{H}\left(\tilde{\mathbf{H}} \tilde{\mathbf{H}}^{H}\right)^{-1},
$$

where the scaling factor $f$ is to ensure the power constraint $\left\|\mathbf{F}_{R F} \mathbf{F}_{B B}\right\|_{F}^{2}=K$.

Remark: While we assume that a simple $\mathrm{ZF}$ precoding is applied in the baseband, other precoding schemes or transmit beamforming techniques can be efficiently applied upon the effective low-dimensional analog channel $\tilde{\mathbf{H}}$.

\section{Complexity and Performance Metrics}

\section{A. Complexity}

It can be observed that the complexity of the proposed schemes is dominated by the calculations of $\tilde{\mathbf{H}}(k, k)$ and $\mathbf{t}_{k}^{i}=\mathbf{H}_{k} \mathbf{p}_{k}^{i}$. Note that $\mathbf{H}_{k} \in \mathcal{C}^{N_{r}^{k} \times N_{t}}$ and $\mathbf{p}_{k}^{i} \in \mathcal{C}^{N_{t} \times 1}$, and therefore the calculation of $\mathbf{q}_{k}^{i}$ in (6) involves a $N_{r}^{k} N_{t}$ multiplications. The calculation of $\tilde{\mathbf{H}}(k, k)$ in Algorithm 1 involves $\left(N_{r}^{k}+N_{r}^{k} N_{t}\right)$ multiplications. This has to be calculated $N_{r}^{k}$ times for each user, both at the transmitter and the receiver, which for the fully-connected system gives (11):

$$
C_{\text {Joint }}=2 \sum_{k=1}^{K} N_{r}^{k}\left(N_{r}^{k}+2 N_{r}^{k} N_{t}\right) .
$$

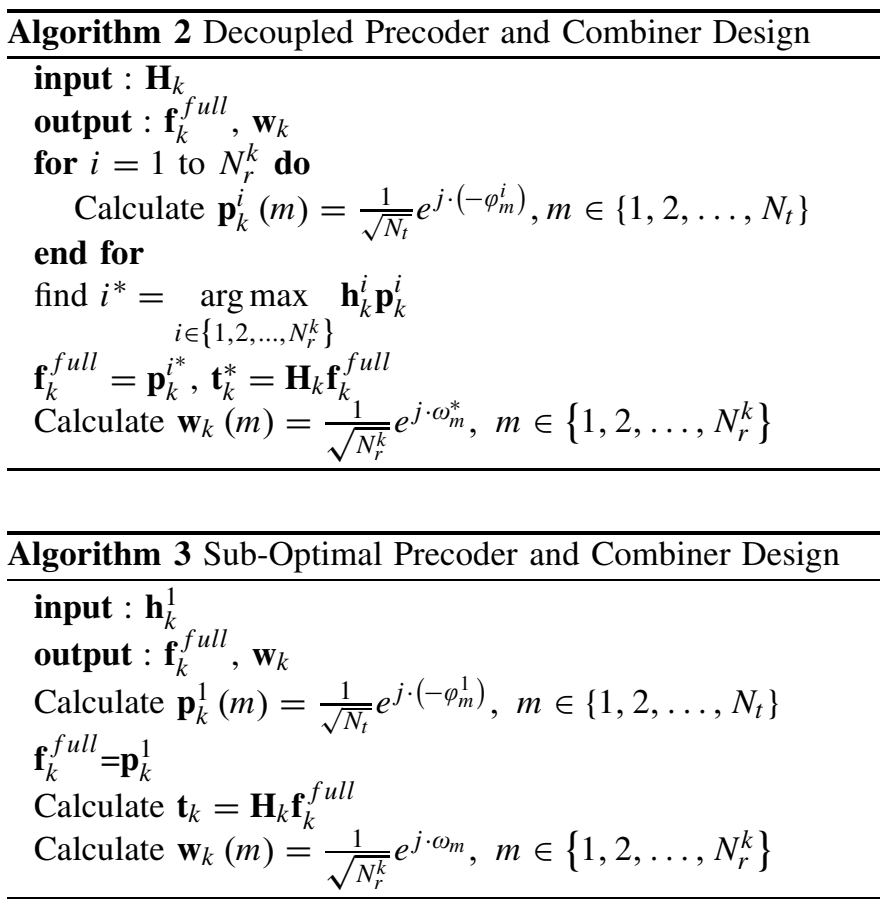

The complexity of Algorithm 2, 3 can be similarly derived as

$$
\begin{aligned}
C_{\text {decoupled }} & =2 \sum_{k=1}^{K} N_{r}^{k}\left(N_{r}^{k} N_{t}\right)+\sum_{k=1}^{K} N_{r}^{k}, \\
C_{\text {sub-optimal }} & =\sum_{k=1}^{K} N_{r}^{k} N_{t} .
\end{aligned}
$$

The complexity for sub-connected structures can be efficiently obtained by substituting $N_{t}$ with $M$ in (11)-(13).

\section{B. Achievable Sum Rate and Energy Efficiency}

By assuming Gaussian input, the received signal-tointerference-plus-noise-ratio (SINR) for $k$ th user can be obtained as

$$
\gamma_{k}=\frac{\frac{P}{K}\left\|\mathbf{w}_{k}^{H} \mathbf{H}_{k} \mathbf{F}_{R F} \mathbf{f}_{B B_{k}}\right\|_{F}^{2}}{\sum_{j \neq k} \frac{P}{K}\left\|\mathbf{w}_{k}^{H} \mathbf{H}_{k} \mathbf{F}_{R F} \mathbf{f}_{B B_{j}}\right\|_{F}^{2}+\sigma^{2}},
$$

and the achievable sum rate is given as $R=\sum_{k=1}^{K} \log _{2}\left(1+\gamma_{k}\right)$. The associated power consumption can be expressed as [12]

$$
\begin{aligned}
P_{\text {full }}= & N_{t}\left(N_{R F}^{t}+1\right) P_{P A}+N_{t} N_{R F}^{t} P_{P S}+P_{B B} \\
& +N_{R F}^{t}\left(P_{R F C}+P_{D A C}\right), \\
P_{\text {sub }}= & N_{t} P_{P A}+N_{t} P_{P S}+P_{B B}+N_{R F}^{t}\left(P_{R F C}+P_{D A C}\right),
\end{aligned}
$$

where $P_{P A}$ denotes the power for the power amplifiers, $P_{P S}$ the power for the phase shifters, $P_{R F C}$ the power for the RF chains, $P_{D A C}$ the power for the digital-to-analog converters, and $P_{B B}$ the power for the baseband processing. The typical value of each component follows [12]. Then, the energy efficiency can be obtained as $\eta=R / P_{\text {full } / s u b}$. 


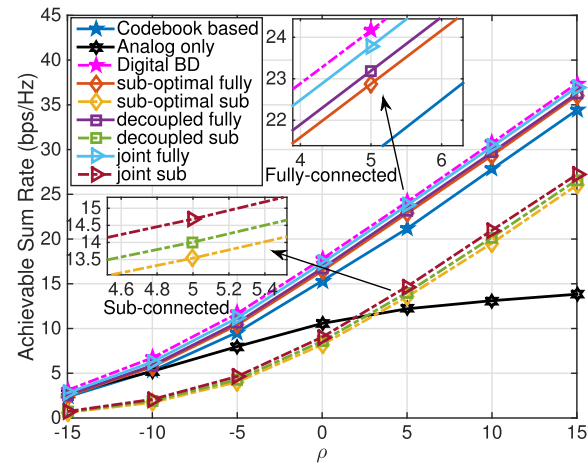

Fig. 2. Sum rate, $N_{t}=64, K=4, N_{r}^{0}=4, L_{u}=6$.

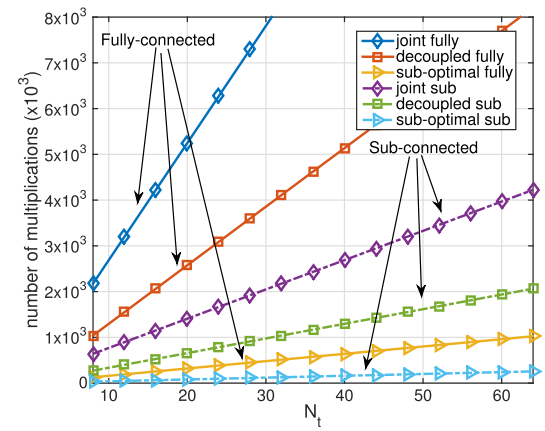

Fig. 3. Complexity, $K=4, N_{r}^{0}=4$.

\section{Numerical Results}

In this section, the numerical results based on Monte Carlo simulations are presented where we employ the limited scattering mmWave channel model in [8] and [9]. Without loss of generality, a uniform planar array (UPA) with the antenna spacing $d=\frac{\lambda}{2}$ is assumed, and the number of propagation paths $L_{u}=6$. We assume the azimuth angles $\theta \in[0,2 \pi]$, and the elevation angles $\phi \in\left[-\frac{\pi}{2}, \frac{\pi}{2}\right]$. For simplicity we assume $N_{r}^{k}=N_{r}^{0}, \forall k$. We compare the proposed schemes with existing codebook based scheme [8], analog beamforming, and unconstrained digital block diagonalization (BD) scheme [13] in different SNR regimes. The SNR in each figure is defined as $\frac{1}{K \sigma^{2}}$, where we assume the total transmit power $P=1$.

Fig. 2 presents the achievable sum rate of different schemes vs. SNR, where the the quantization of the azimuth and elevation angle is 4 bits and 3 bits respectively for the codebook based scheme. All of the proposed schemes outperform the existing codebook based scheme and analog beamforming, and can approach the performance of digital BD with an SNR loss less than 1dB. A similar trend can be observed for the sub-connected structure, where there is a loss in sum rates as only part of the array gain can be obtained. While not shown, the performance gain of the proposed schemes over codebook based scheme becomes larger with the increase in $L_{u}$.

Fig. 3 and Fig. 4 compare the complexity and the energy efficiency for fully- and sub-connected structures. Less complexity is needed for sub-connected structures, and the energy efficiency of sub-connected structures is much higher due to the reduced number of phase shifters required, which makes them more favourable for practical implementation. The joint approach with sub-connected structures achieves a higher

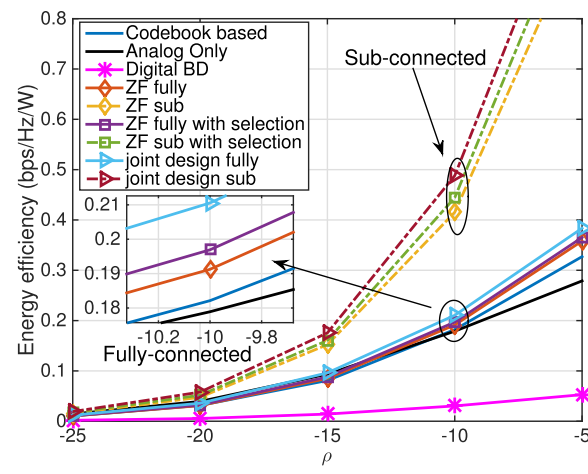

Fig. 4. Energy efficiency, $N_{t}=64, K=4, N_{r}^{0}=4, L_{u}=6$.

energy efficiency, due to its favourable performance to power consumption tradeoff.

\section{CONCLUSION}

In this letter, we propose three low-complexity hybrid precoding and combining designs for mmWave MU-MIMO systems, for both fully-connected and sub-connected structures. By combining a virtual path selection in the RF domain with a low-dimensional baseband precoder, the proposed schemes provide a much improved performance-complexity trade-off compared to their conventional counterparts.

\section{REFERENCES}

[1] Z. Pi and F. Khan, "An introduction to millimeter-wave mobile broadband systems," IEEE Commun. Mag., vol. 49, no. 6, pp. 101-107, Jun. 2011.

[2] T. S. Rappaport et al., "Millimeter wave mobile communications for 5G cellular: It will work!" IEEE Access, vol. 1, pp. 335-349, May 2013.

[3] F. Rusek et al., "Scaling up MIMO: Opportunities and challenges with very large arrays," IEEE Signal Process. Mag., vol. 30, no. 1, pp. 40-60, Jan. 2013.

[4] V. Venkateswaran and A. van der Veen, "Analog beamforming in MIMO communications with phase shift networks and online channel estimation," IEEE Trans. Signal Process., vol. 58, no. 8, pp. 4131-4143, Aug. 2010.

[5] F. Gholam, J. Via, and I. Santamaria, "Beamforming design for simplified analog antenna combining architectures," IEEE Trans. Veh. Tech., vol. 60, no. 5, pp. 2373-2378, Apr. 2011.

[6] O. El Ayach, S. Rajagopal, S. Abu-Surra, Z. Pi, and R. W. Heath, Jr., "Spatially sparse precoding in millimeter wave MIMO systems," IEEE Trans. Wireless Commun., vol. 13, no. 3, pp. 1499-1513, Mar. 2014.

[7] C. E. Chen, "An iterative hybrid transceiver design algorithm for millimeter wave MIMO systems," IEEE Wireless Commun. Lett., vol. 4, no. 3, pp. 285-288, Jun. 2015.

[8] A. Alkhateeb, G. Leus, and R. W. Heath, Jr., "Limited feedback hybrid precoding for multi-user millimeter wave systems," IEEE Trans. Wireless Commun., vol. 14, no. 11, pp. 6481-6494, Nov. 2015.

[9] D. H. N. Nguyen, L. B. Le, and T. Le-Ngoc, "Hybrid MMSE precoding for mmWave multiuser MIMO systems," in Proc. IEEE ICC, Kuala Lumpur, Malaysia, May 2016, pp. 1-6.

[10] L. Liang, W. Xu, and X. Dong, "Low-complexity hybrid precoding in massive multiuser MIMO systems," IEEE Wireless Commun. Lett., vol. 3, no. 6, pp. 653-656, Dec. 2014.

[11] A. Garcia-Rodriguez, V. Venkateswaran, P. Rulikowski, and C. Masouros, "Hybrid analog-digital precoding revisited under realistic RF modeling," IEEE Wireless Commun. Lett., vol. 5, no. 5, pp. 528-531, Oct. 2016.

[12] R. Méndez-Rial, C. Rusu, N. González-Prelcic, A. Alkhateeb, and R. W. Heath, Jr., "Hybrid MIMO architectures for millimeter wave communications: Phase shifters or switches?" IEEE Access, vol. 4, pp. 247-267, Jan. 2016.

[13] Q. H. Spencer, A. L. Swindlehurst, and M. Haardt, "Zero-forcing methods for downlink spatial multiplexing in multiuser MIMO channels," IEEE Trans. Signal Process., vol. 52, no. 2, pp. 461-471, Feb. 2004. 